BULGARIAN ACADEMY OF SCIENCES

CYBERNETICS AND INFORMATION TECHNOLOGIES • Volume 15, No 6

Special Issue on Logistics, Informatics and Service Science

Sofia $\bullet 2015$

Print ISSN: 1311-9702; Online ISSN: 1314-4081

DOI: $10.1515 /$ cait-2015-0065

\title{
Three-Dimensional Image Measurement by Pattern Projection Using a Single Observation Image
}

\author{
Ke Sun ${ }^{1}$, Cunwei $L u^{2}$ \\ ${ }^{1}$ Guilin University of Electronic Technology, Guilin, 541004 China \\ ${ }^{2}$ Fukuoka Institute of Technology 3-30-1 Wajirohigashi, Higashi-ku, Fukuoka 811-0295, Japan \\ Email: sunke2015@guet.edu.cn
}

\begin{abstract}
Since three-dimensional image measurement allows object surface shapes and dimensions to be obtained quickly and without any contact, it has recently been intensively studied in a wide range of fields, including industry, medicine and security. Three-dimensional image measurement technologies can be broadly classified into passive techniques, such as stereovision and active techniques, such as patterned light projection. Among these, the method of projecting optimum intensity modulated light patterns for three-dimensional image measurement can obtain three-dimensional information on the measured object with a single projection, so it is expected to be highly applicable in practice. Measurement can be performed using a single observation image when the object to be measured has simple colouration or surface reflectivity, but for complex objects, eliminating the influence of colour and surface reflectivity requires a reference image to correct the intensity of the observed pattern. To address this, we propose an analysis method and image correction technology, using a novel colour system for realizing threedimensional measurements using only one observation image.
\end{abstract}

Keywords: Three-dimensional shape measurement, optimal intensity-modulation pattern projection.

\section{Introduction}

The improvement of the theoretical methods for practical application of threedimensional image measurement technologies has been studied. Methods of threedimensional image measurement can be roughly divided into active and passive methods [1]. Passive measurement methods, such as stereovision, using two cameras are suitable for measuring object contours, but are difficult to be applied to the measurement of full object surfaces or to the surface measurement of largely 
featureless objects. In contrast, active measurement methods, such as patterned light projection can be applied to surface measurements of featureless objects, but the measurement requires multiple projections of the patterned light onto the measured object, not only increasing the measurement times, but also making the measurement of non-stationary objects difficult [2].

Previous research has proposed methods of projecting optimum intensity modulated light patterns for three-dimensional image measurement (henceforth referred to as "optimal pattern projection measurement") with the goal of shortening the amount of time needed for measurement and improving the measurement accuracy [3]. Optimal pattern projection measurement is a method of projecting a pattern of light onto a measured object with optimized intensity changes and analyzing the acquired pattern images. For measured objects with surfaces having a simple colour distribution, the optimal pattern projection methods can in principle obtain three dimensional information regarding the object surfaces from a single image [4-6]. Cases, where the colour distribution or surface reflection are complex, require capturing an overall illuminated image, in which the patterned light is not projected for use as a reference image; the effects of surface reflection can be eliminated through division with the observed pattern image. This allows threedimensional measurement with a single projection and two captures, but when measuring a moving image, this second capture disassociates the correspondence between the two images, making it difficult to accurately correct the pattern intensities and thus perform measurements.

The aim of this research is realizing three-dimensional measurement, using a single light pattern projection and imaging. To achieve this, an image analysis technique is proposed that does not rely on a repeated image capture, applying instead a method of image analysis to create an overall illuminated image from the initial observed pattern image. Specifically, we propose a novel Intensity/Hue/Contrast (IHC) colour system using a novel regional segmentation technique to partition the observed pattern image into striped and non-striped regions. The colour space is then partitioned into a number of subspaces and the observed pattern image is partitioned into a number of sub-images in each colour subspace. A saturation quota basis is also proposed where in each sub-image, pixels in the striped regions are interpolated using pixels in the non-striped regions that fulfill the saturation quota basis to generate the reference image. Finally, the reference images generated in each colour space are combined to generate the fully illuminated image. Thus, a three-dimensional measurement is realized using a fully illuminated image generated from a single captured observed pattern image.

To improve the stripe detection accuracy of the patterned light projection measurement technology, a Neutral Density-Low-Pass Filter (ND-LPF) analysis method is proposed, based on frequency analysis, and an intensity correction method is proposed for the observed pattern image using an ND-LPF.

The proposed method comprises six steps. Step 1 projects the optimum intensity modulation pattern onto the measurement target. The object of measurement onto which the patterned light is projected is then photographed and taken as an observed pattern image $I_{(1)}$. Step 2 extracts the observed pattern, which 
is taken as an extracted pattern image $I_{(2)}$. Step 3 uses a fully illuminated image $I_{(3)}$ for non-division pattern correction. Step 4 divides the extracted pattern image and the generated fully illuminated image to correct the intensity values, and filters the frequency domains in the corrected pattern image $I_{(4)}$ to re-correct the pattern intensity values. Step 5 detects the correspondence between the observed pattern and the projected pattern. Step 6 calculates the three-dimensional information of the measured object based on triangulation from the detected correspondence. This paper describes Steps 2 through 4. Fig. 1 shows the image relationships at each step.

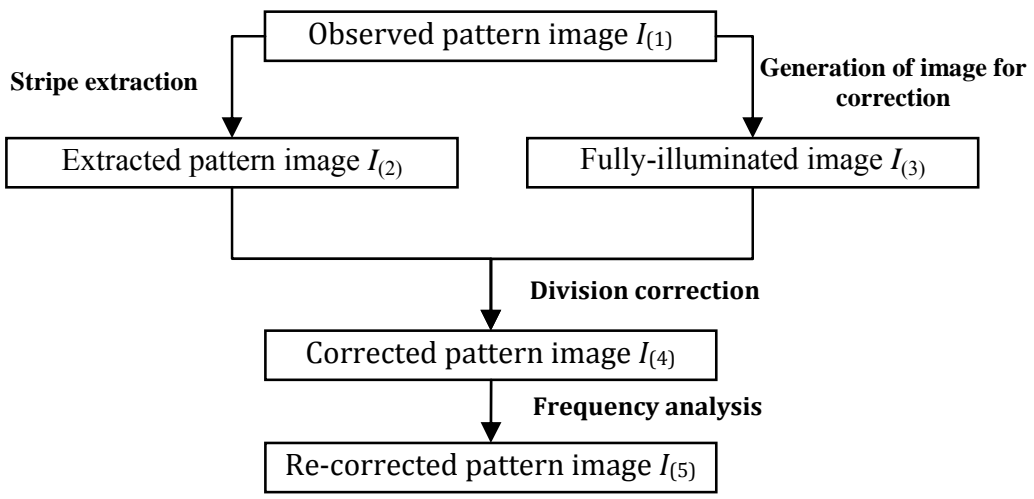

Fig. 1. Image relations at each step of the process

\section{Measurement principle}

\subsection{Colour system}

The central problem of this study is generating the fully illuminated image. The fully illuminated image in the existing division correction methods is an image captured with no projected pattern. In this study however, the fully illuminated image is not captured; the stripes in the projected pattern are removed from the observed pattern image to create a fully illuminated image that can represent the original pattern of the measured object.

Generating a fully illuminated image requires the construction of a colour system. The colour system for image measurement in this study requires three elements. First, to make easier the distinguishing between the regions in the observation image, in which the pattern is projected onto the measured object surface and those regions where it is not, we can quantitatively, accurately and simply calculate the change in the intensity on the measured object surface due to the projected pattern. To do so, an intensity value measurement method is proposed that combines the maximal values $I_{\max }$ and mean values $I_{\text {mean }}$ of the intensity value in each RGB channel. To avoid the influence of the surface colour distribution on the measurement target and to accurately extract the stripes, a specific method is used from literature that applies the image intensity values for $I_{\max } . I_{\text {mean }}$ is used to correct the observed pattern intensities in order to avoid the effects of noise and to obtain a stable pattern [5]. 
The second required element is a quantitative and accurate representation of the diversity of surface colouration on the measured object, as seen in the observation image, so that various surface colours can be distinguished. To reduce the effects of the measured object colouration on the measurement results, a colour system is introduced with a formula for calculating the hue values in HLS colour space related to colour information [7-9].

Finally, commonalities must be determined between regions in the observation image in which the pattern is projected onto the measured object surface and those where it is not, so that invariance can be accurately represented even when the projected pattern impinges upon the measured object surface. Since chroma affects the variation in colour saturation due to illumination by the light pattern, a chroma system is introduced [7]. Chroma value calculations in the present research are characterized by considering pixels with the same saturation values as having the same reflection characteristics. Therefore, a robust calculation method is proposed with a symmetric RGB that retains invariable chroma values, both when the measured object is illuminated with the patterned light and when it is not. In the ideal case, the reflectivity of the pixels in the observed pattern image in a same hue region will have the same chroma, so the positional exchanges of such pixels are considered as not changing the image. Therefore, pixels in regions illuminated by the pattered light are exchanged with pixels in regions not illuminated by the patterned light, but having the same hue and saturation values to reconstruct the fully illuminated image with no projected pattern.

A colour space is proposed based on the hue, saturation and image intensity for pattern image measurement. For the actual system, we propose intensity $I$, hue $H$, and chroma $C$ equations as the following:

$$
I:\left\{\begin{array}{l}
I_{\max }(x, y)=\max \left\{I_{R}(x, y), I_{G}(x, y), I_{B}(x, y)\right\}, \\
I_{\text {mean }}(x, y)=\frac{1}{3}\left[I_{R}(x, y)+I_{G}(x, y)+I_{B}(x, y)\right],
\end{array}\right.
$$

$$
C:\left\{\begin{array}{c}
H: H(x, y)=\arctan \frac{\sqrt{3}\left[I_{G}(x, y)-I_{B}(x, y)\right]}{\left[I_{R}(x, y)-I_{B}(x, y)\right]+\left[I_{R}(x, y)-I_{G}(x, y)\right]}, \\
C_{1}=\frac{\left[I_{R}(x, y)-I_{B}(x, y)\right]^{2}}{\left[I_{R}(x, y)-I_{B}(x, y)\right]^{2}+\left[I_{R}(x, y)-I_{G}(x, y)\right]^{2}+\left[I_{G}(x, y)-I_{B}(x, y)\right]^{2}}, \\
C_{3}=\frac{\left[I_{R}(x, y)-I_{G}(x, y)\right]^{2}}{\left[I_{R}(x, y)-I_{B}(x, y)\right]^{2}+\left[I_{R}(x, y)-I_{G}(x, y)\right]^{2}+\left[I_{G}(x, y)-I_{B}(x, y)\right]^{2}}, \\
{\left[I_{R}(x, y)-I_{B}(x, y)\right]^{2}+\left[I_{R}(x, y)-I_{G}(x, y)\right]^{2}+\left[I_{G}(x, y)-I_{B}(x, y)\right]^{2}}
\end{array} .\right.
$$

Here $(x, y)$ is the coordinate of a target pixel, and $I_{R}(x, y), I_{G}(x, y)$ and $I_{B}(x, y)$ are the respective intensity values in the RGB channels of the pixel. $I_{\max }(x, y)$ is the maximum intensity value in each channel of the pixel of interest, and $I_{\text {mean }}(x, y)$ is the mean intensity value in the RGB channels of the pixel of interest. $H(x, y)$ is the hue value of the pixel of interest. $C_{1}(x, y), C_{2}(x, y)$ and $C_{3}(x, y)$ are the saturation values of the pixel of interest. 


\subsection{Stripe extraction and region division of the observed pattern image}

This section explains the principles of Step 2. To correctly detect the location of the stripe from the observed pattern image, it is necessary to extract the position and the intensity of stripes in the observed pattern. Methods for extracting contours generally use binarization or differentiation, but as described below such methods are not highly stable for extracting stripes from intensity modulation patterns.

Fig. 2 shows a pattern projected onto the surface of a measured object. For the observed intensity modulation pattern image, due to the influence of the surface colour and reflection of the measuring object, as shown in the figure, the intensity values in the areas onto which the pattern is projected will not necessarily be higher than those in the regions onto which the pattern is not projected. They will often be higher or lower, depending on the location. Using an ordinary fixed threshold when extracting pattern stripes can thus easily result in erroneous extractions, making the stable stripe extraction difficult. This is another weakness in binarization as a contour extraction method. Further, the intensity distribution of the intensity modulation pattern stripes is similar to a cosine distribution (Fig. 2b). Consequently, when the first derivative gradient and the second derivative Laplacian are used, the contour edges are extracted along with edges due to different colouration, rather than only the pattern stripes being extracted, thus making it difficult to apply general differentiation methods.

The intensity distribution of the stripes changes with frequency, so a method of pattern extraction is proposed which uses dynamic local thresholds based on the direction of the intensity change in the characteristics of the pattern stripes.

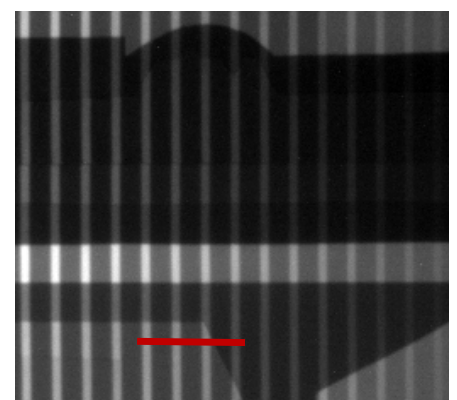

(a)

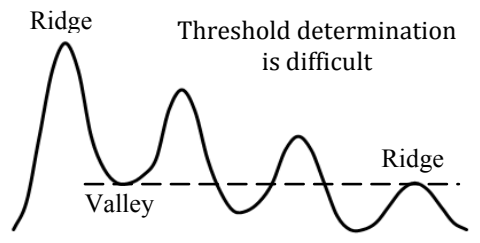

(b)

Fig. 2. Stripe intensity distribution in the observed pattern: Sample observed pattern image (a); intensity distribution along the red line in (a) (b)

The observed pattern image is scanned in a direction perpendicular to the stripes, using the next Equation to determine the threshold for binary processing of the pixel of interest $(x, y)$ :

$$
\operatorname{Th}(x, y)=\operatorname{Th}_{0}+\frac{1}{2 a+1} \sum_{k=-a}^{a} I_{\max }(x, y+k),
$$

where $a$ is the filter size determined by the width of the projected pattern stripes, $\mathrm{Th}_{0}$ is the initial threshold, and $\operatorname{Th}(x, y)$ is a threshold function.

The threshold value is next used to binarize the intensity values of the pixels of interest following equation: 


$$
\left\{\begin{array}{lll}
I(x, y)=I_{\text {mean }}(x, y) & \text { if } \quad I_{\text {max }}(x, y) \geq \operatorname{Th}(x, y), \\
I(x, y)=0 & \text { if } \quad I_{\text {max }}(x, y)<\operatorname{Th}(x, y) .
\end{array}\right.
$$

On the basis of the above calculation, the areas where $I(x, y) \neq 0$ are defined as stripe regions, and the areas where $I(x, y)=0$ as non-stripe regions. In the stripe regions the stripes of the projected pattern are projected, and in the non-stripe regions the stripes of the projected patterns are not projected.

This enables the extraction of stripes in the projected pattern from the observed pattern image, allowing the observed pattern image to be partitioned into stripe regions and non-stripe regions. We refer to this as an extracted pattern image $I_{(2)}$.

\subsection{Generation of a fully illuminated image}

This section explains the principles of Step 3. The basic idea for generating a fully illuminated image is using information from pixels in non-stripe regions to interpolate the colour intensity values of pixels in the stripe regions, thereby generating an entire illumination image. This is possible because the continuity of the colour intensity information on the object surface gives an approximation of the hue information for stripe and non-stripe regions. The colour intensity space is therefore partitioned into $N$ equal parts by the hue value, and the non-stripe region pixels can be searched for within each colour intensity subspace to interpolate stripe areas.

Specifically, we first use Equation (6) below to partition the colour intensity space into $N$ colour intensity subspaces according to the value of hue $H$. The range of the hue values in each colour intensity subspace $N$ is taken to be $[2(n-1) \pi / N$, $2 n \pi / N]$. For example, letting $N=16$ partitions the colour intensity space into 16 equal parts, so the hue range of the colour intensity subspace is $22.5^{\circ}$. Furthermore, the hue range $H_{1}$ in the colour intensity subspace $I_{m, 1}$ is $\left[0^{\circ}, 22.5^{\circ}\right]$, the hue range $\mathrm{H}_{2}$ in the colour intensity subspace $I_{m, 2}$ is $\left[22.5^{\circ}, 45^{\circ}\right]$, and the hue range $H_{16}$ in the colour intensity subspace $I_{m, 16}$ is $\left[337.5^{\circ}, 360^{\circ}\right]$ :

$$
I_{m, n}(x, y)=\left\{\begin{array}{cc}
I_{m, n}(x, y) & \text { if } \frac{2(n-1) \pi}{N} \leq H_{n}(x, y)<\frac{2 n \pi}{N}, m \in R, G, B, n=1,2, \ldots, N, \\
0 & \text { otherwise. }
\end{array}\right.
$$

We next calculate the hue value $H(x, y)$ for each pixel $P(x, y)$ in the observed pattern image, and if $H(x, y) \in H_{n}$ we take the pixel $P(x, y)$ as a pixel in the colour intensity subspace $I_{m, N}(x, y)$. In this way we partition the observed pattern image into $N$ sub-images. As an example, Fig. 3 shows the results of dividing an observed pattern image into 16 sub-images.

We further perform the above-described stripe extraction on each sub-image, and use RGB information from the non-stripe region pixels to interpolate RGB values of pixels in the stripe regions.

First, we set a space filter $M(X \times Y)$ centered on a pixel of interest in the striped region (referred to below as a "stripe pixel of interest") in each sub-image. 

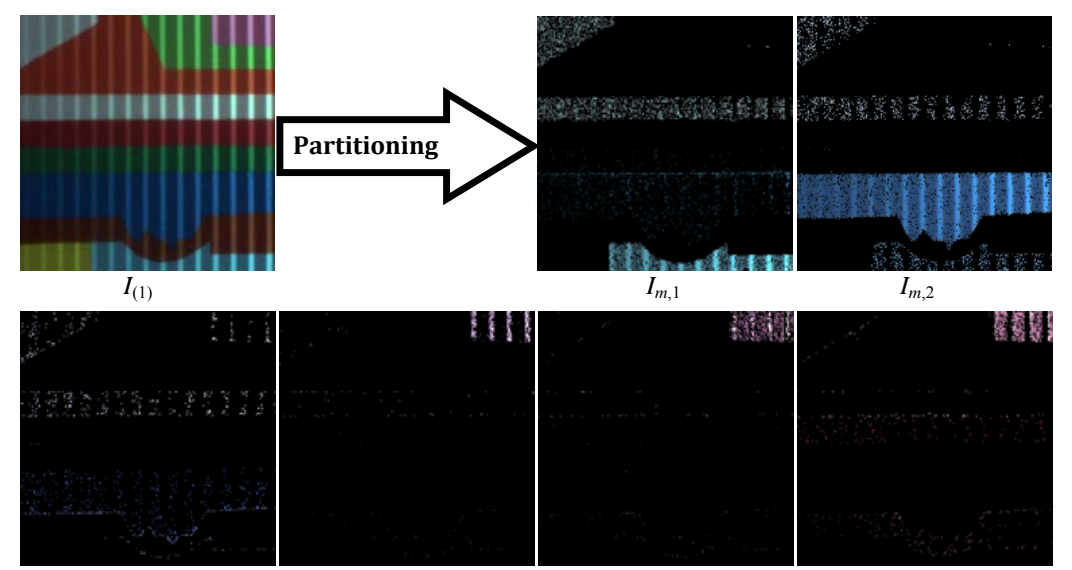

$I_{m, 3}$ $I_{m, 4}$ $I_{m, 5}$ $I_{m, 6}$
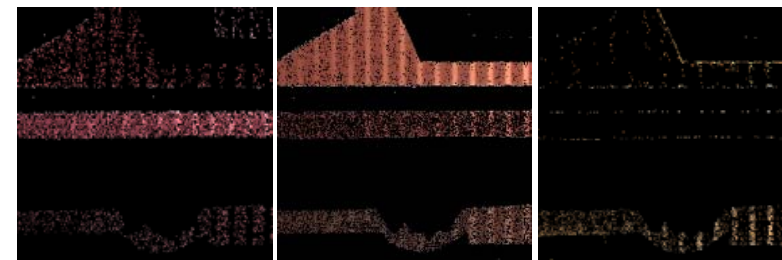

$I_{m, 7}$

$I_{m, 8}$

$I_{m, 9}$

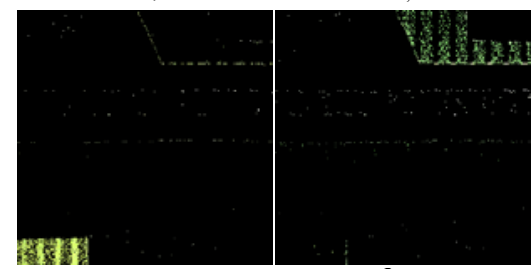

$I_{m, 1}$

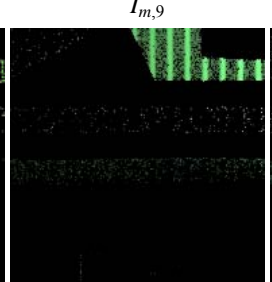

$I_{m, 13}$

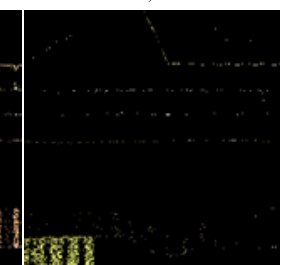

$I_{m, 10}$

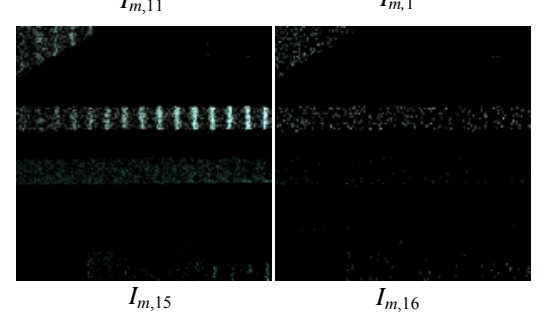

Fig. 3. Observed pattern image $I_{(1)}$ and the partitioned sub-image $I_{m, k}, k=1,2, \ldots, 16$

We will explain the interpolation process based on the example in Fig. 4. Fig. 4a shows a part of the blue sub-image for Fig. 3 and an enlarged view of the area within the blue dotted line. Fig. 4b shows the striped region (left) and the nonstriped region (right) in the enlarged portion. Fig. $4 \mathrm{c}$ is an image of the intensity distribution of each pixel within a filter $M$, and Fig. $4 d$ shows the use of the stripe pixel of interest to calculate the saturation quotas for each non-stripe pixel in the filter. This calculation uses next equations:

$$
\left\|C_{l}\left(x^{\prime}, y^{\prime}\right)\right\|=\left[C_{l}(x, y)-C_{l}\left(x^{\prime}, y^{\prime}\right)\right]_{(x, y),\left(x^{\prime}, y^{\prime}\right) \in M}^{2}, \quad l=1,2,3,
$$




$$
\operatorname{norm}\left(x^{\prime}, y^{\prime}\right)=\sum_{l=1}^{3}\left\|C_{l}\left(x^{\prime}, y^{\prime}\right)\right\| .
$$

Fig. $4 \mathrm{e}$ shows the selection of the non-stripe pixel with the smallest saturation quota for the stripe pixel of interest and the use of the next equation to exchange the colour intensity information of the stripe pixel of interest:

$$
I_{R, G, B}(x, y)=I_{R, G, B}\left(x^{\prime}, y^{\prime}\right) \text { if } \min _{\left(x^{\prime}, y^{\prime}\right) \in M}\left\{\operatorname{norm}\left(x^{\prime}, y^{\prime}\right)\right\} .
$$

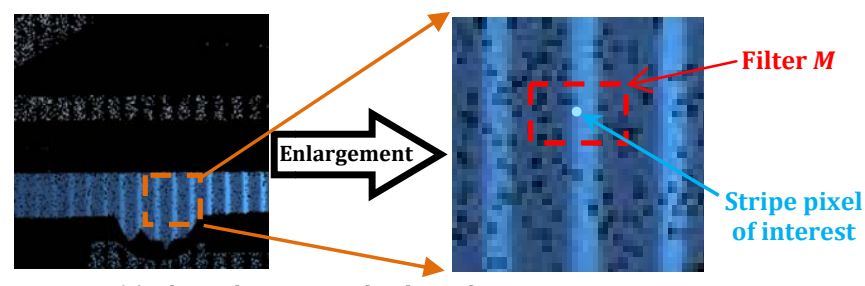

(a) Blue sub-image and enlarged portion
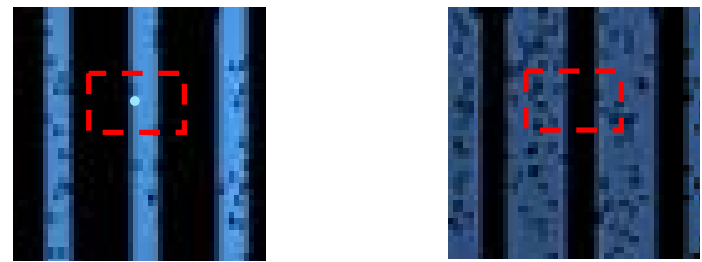

(b) Stripe and non-stripe regions in the enlarged portion

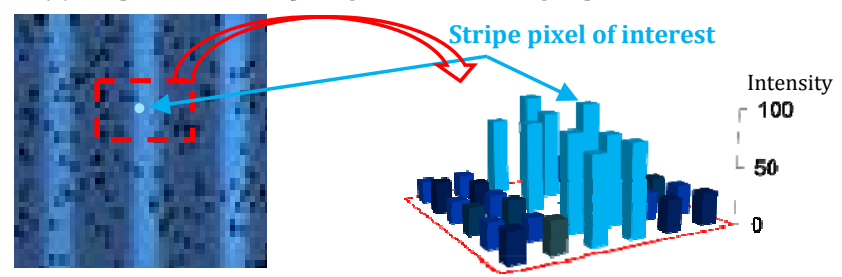

(c) Pixel distribution in the enlarged portion and within $M$

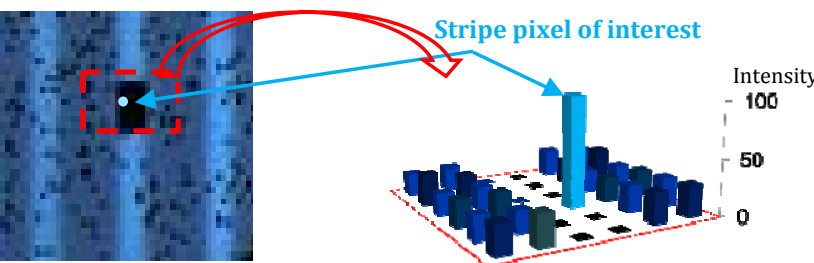

(d) Stripe pixel of interest in the enlarged portion and within $M$

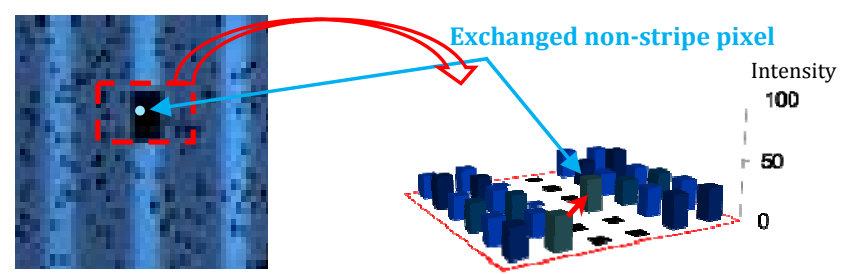

(e) Swapping stripe pixels selected from the stripe image

Fig. 4. Stripe region pixel interpolation 
Processing is complete when all stripe pixels in each sub-image are interpolated, and 16 complementary sub-images are obtained.

Finally, as Fig. 5 shows, we add the colour intensity information to pixels corresponding to image coordinates in the 16 complementary sub-images, thereby generating the single fully illuminated image $I_{(3)}$.
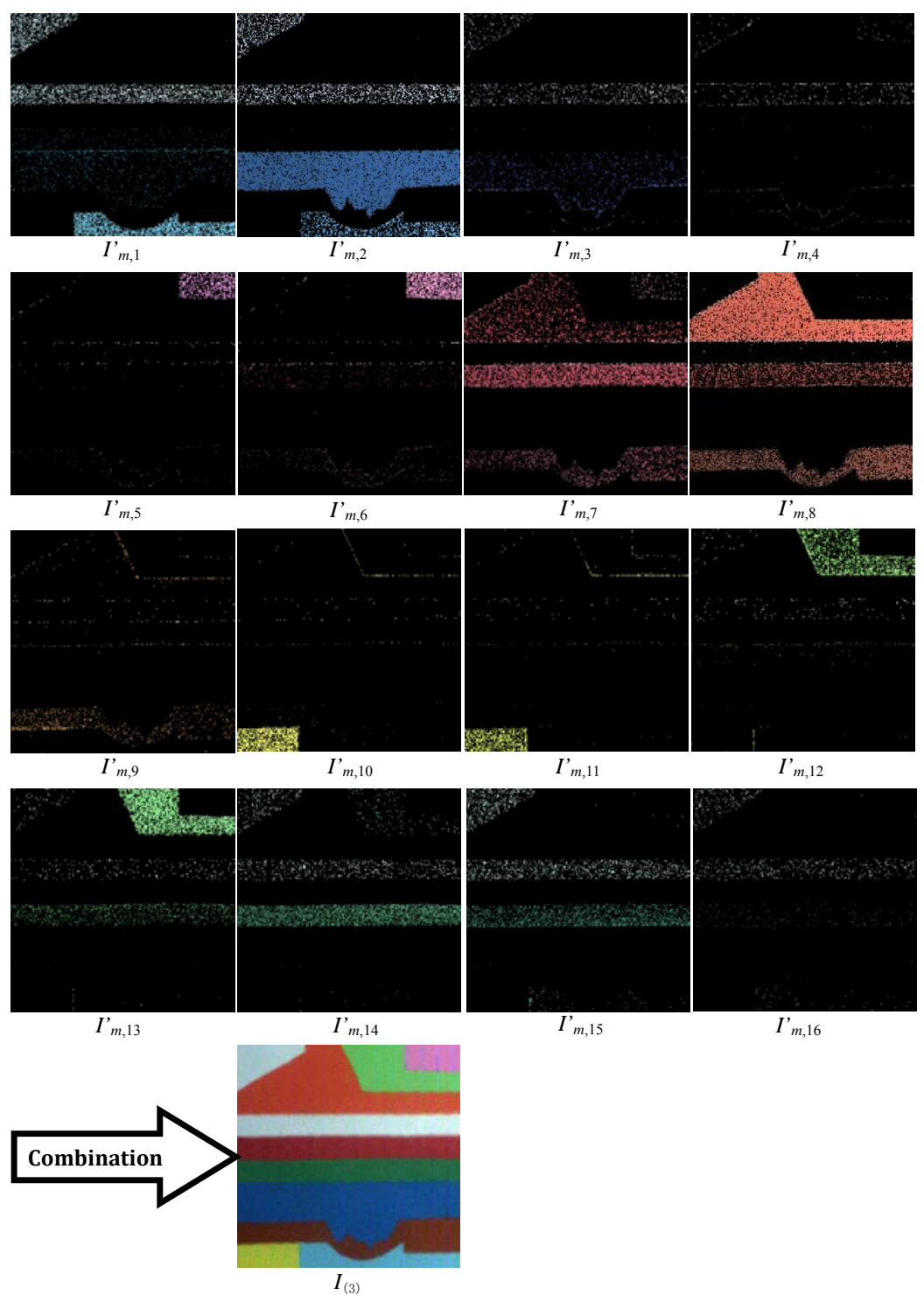

$I_{m, 16}^{\prime}$

Fig. 5. Interpolated sub-images $I_{m, k}, k=1,2, \ldots, 16$, generated from the fully illuminated image $I_{(3)}$

2.4. Correction of the stripe intensity in the observed pattern

This section describes the principle of the method used in Step 4. Intensity correction of the observed pattern stripes is performed using division correction and 
two types of correction in the frequency domains. Division correction uses the extracted pattern image $I_{(2)}$, and the method of the next equation to obtain the corrected pattern image $I_{(4)}[10]$ :

$$
I_{(4)}(x, y)=K_{0} \frac{I_{(2)}(x, y)}{I_{(3)}(x, y)} .
$$

Here $K_{0}$ is the intensity of the ambient light.

The correction in the frequency domain shows some high-frequency noise as compared to the created fully illuminated image. For this reason, the influence of the measured object surface colour is significantly reduced after division correction, but some surface colour may remain. Therefore, a method must be established for further correction in the frequency domain of the pattern image after the division correction [11-13].

The basic idea of this correction in the frequency domain is the existence of intensity edges in the feature-quantity power spectral image having light-dark patterns similar to the observed pattern image. Extracting these intensity edges allows the obtaining of clean pattern stripes. However, the high frequencies must be removed smoothly to prevent the Gibbs phenomenon. Therefore, the use of an ND-LPF filter, such as the one shown in the next equation is proposed:

$$
f(u, v)=\frac{1}{1.0+(\sqrt{2}-1) p^{1 / 4}},
$$

where $(u, v)$ are coordinates in the power spectrum space, $F(u, v)$ is the filter coefficient, and $p$ is the radius of the filter at the center of the power spectrum images. The units are in pixels.

Using the proposed method, the relationship between the resulting images is expressed by the following equation:

$$
F_{(4)}=F\left\{I_{(4)}\right\},
$$

where $F$ is a Fourier transform, and $F_{(4)}$ is the power spectral image of $I_{(4)}$.

The remaining power spectrum after filtering is

$$
F_{(5)}=f(u, v) F_{(4)} \text {. }
$$

Finally,

$$
I_{(5)}=F^{-1}\left\{F_{(5)}\right\},
$$

where $F^{-1}$ is the inverse Fourier transform.

\section{Results of the experiment}

Verification experiments were performed with a system comprised of a generalpurpose floodlight, a digital camera, and a computer (Fig. 6). The camera was placed approximately $105 \mathrm{~cm}$ from the measured object $[1,2,5]$. Fig. 7 a shows the three subjects of the experiment: a large quadrangular cardboard box on the right, a small box at the upper left, and a small box at the lower left. The two boxes on the left are stacked. 


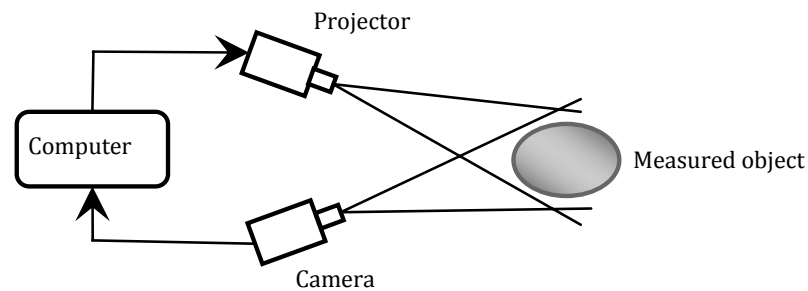

Fig. 6. Experimental system

The dimensions of the large quadrangular box on the right in Fig. 7a were $18 \mathrm{~cm}$ in length, $10 \mathrm{~cm}$ in width, and $29 \mathrm{~cm}$ in height. The dimensions of the small quadrangular box on the left were $8 \mathrm{~cm}$ in length, $7 \mathrm{~cm}$ in width, and $13 \mathrm{~cm}$ in height. Fig. $7 \mathrm{~b}$ shows the twenty stripes in the optimal intensity modulation pattern. The stripes had an average intensity value of 185 , a minimum value of 119 , and maximum value of 252 . The minimum difference of the adjacent stripes was 28 .

The following diagram shows the experimental results at each stage of the process in the proposed method.
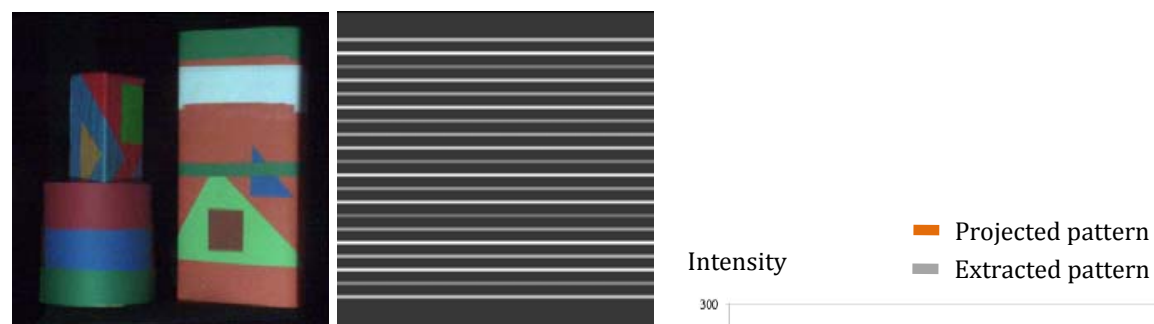

(a) Cardboard box

(b) Optimal intensity modulation pattern for 20 stripes
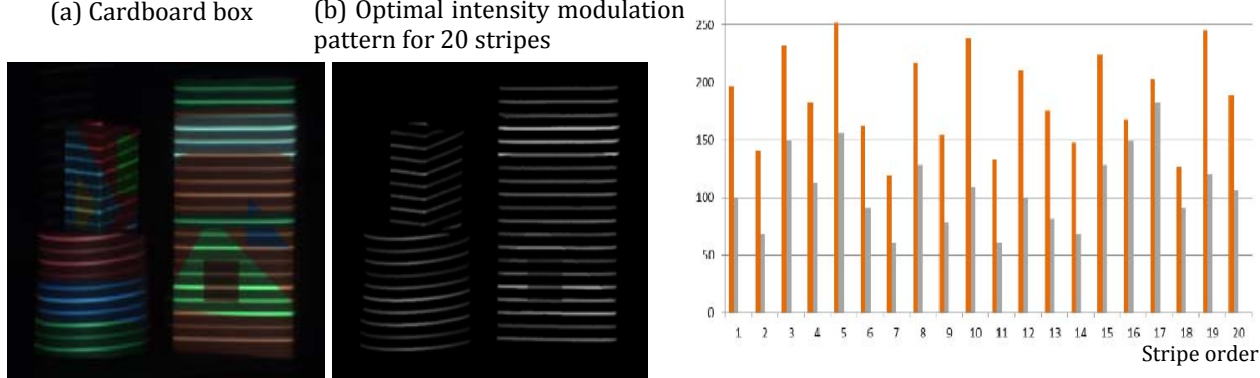

(c) Observed pattern image (d) Extracted pattern image $I_{(1)}$

$I_{(2)}$

(e) Comparison of the projected pattern intensity distribution for Fig. $7 \mathrm{~b}$ and extracted pattern intensity distribution for the right side of Fig. $7 d$

Fig. 7. Results of the cardboard box experiment (1)

Fig. 7c shows the observed pattern image $I_{(1)}$ captured with the twenty stripes of the optimal pattern in Fig. 7a projected onto the combination of boxes in Fig. $7 b$.

Fig. $7 \mathrm{~d}$ shows the extracted pattern image $I_{(2)}$ resulting from the introduced two-step method. As it can be seen in this figure, there are residual influences of the surface colouration of the boxes. Deep colours lower the intensity value of the stripe in this area to the low values in the surrounding stripes, while conversely the 
light colours make the intensity values of the stripes higher, so the regularity of the intensity value changes over the entire pattern is disturbed. Fig. 7e shows the results of comparing the projected pattern in Fig. $7 \mathrm{~b}$ and the intensity distribution of the extracted pattern on the right side of Fig. 7d, with the intensity distribution of the projected pattern (Fig. 7b) in orange and the intensity distribution of the extracted pattern on the right side of Fig. $7 \mathrm{~d}$ in gray. Here the calculated pattern intensity value for a given stripe is taken to be the average value of the pixel intensities in the stripe. This makes the effects of the surface colouration easier to understand. For example, in the pattern on the right side of Fig. $7 \mathrm{~d}$, the fourth, fifth and sixth stripe intensities from the top are shown as the fifteenth, sixteenth, and seventeenth gray lines in Fig. 7e, and due to the white colouration of the measured object surface, the intensities of the sixteenth and seventeenth stripes are higher than that of the fifteenth. In reality, however, according to the pattern of change in the intensity for the stripes (orange), the fifteenth stripe must have higher intensity. In such pattern intensity distribution we can see that it is not possible to construct a correspondence with the projected pattern.

In the processing of Step 3, the non-stripe pixels are used as shown in Fig. 7c to interpolate the stripe regions, thereby creating the fully illuminated image $I_{(3)}$ as shown in Fig. 8a. Comparing this to the captured image, as shown in Fig. 7a, we can see that some noise remains as an after-effect of the pattern on the surface of the measured object. While this will somewhat affect any subsequent intensity corrections, the state before the pattern projection is more or less recovered. In particular, the vividness and colour regions are both accurately restored.

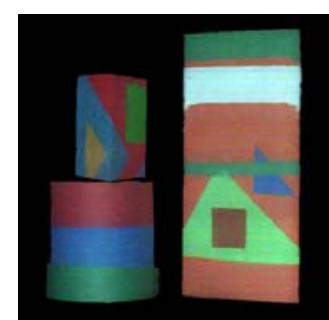

(a) Fully-illuminated image $I_{(3)}$

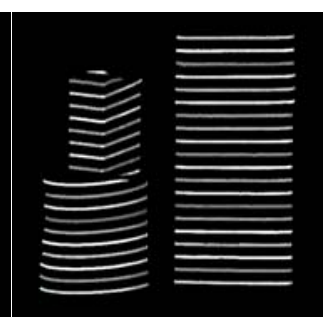

(b) Corrected pattern image $I_{(4)}$

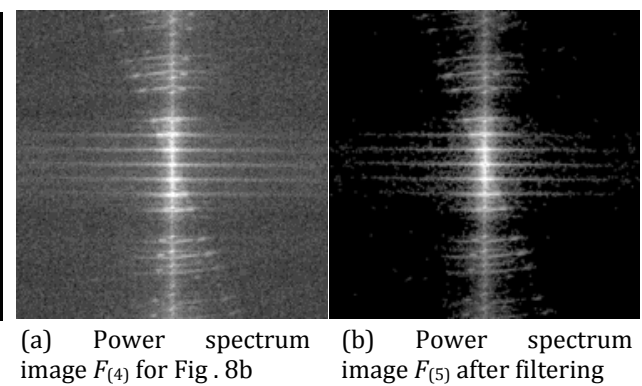

$\begin{array}{ll}\text { Intensity } & \text { Projected pattern } \\ & \text { Corrected pattern }\end{array}$

300

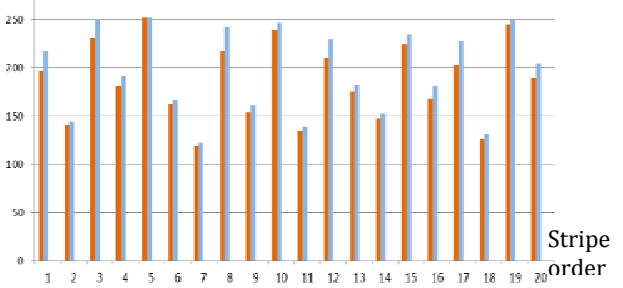

(c) Comparison of the intensity distributions for a projection pattern in Fig. $7 \mathrm{~b}$ and the right side of Fig. 8b

Fig. 8. Results of the cardboard box experiment (2)

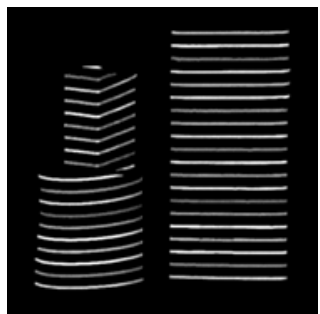

(c) Re-corrected pattern image $I_{(5)}$

Fig. 9. Results of the cardboard box experiment (3) 
Regarding the processing in Step 4, the image in Fig. 8b shows the result of dividing Figs $7 \mathrm{c}$ and $8 \mathrm{a}$ to give the correction pattern image $I_{(4)}$. Comparing this with Fig. 7d, it can be seen that most of the effects of surface reflection have been removed and the pattern intensity has been corrected. However, the intensity distribution of the pixels in each stripe is uneven and some noise remains, so obtaining an ideal observed pattern, such as the projected pattern requires further pattern intensity correction. Fig. 8c compares the intensity distributions for the projected patterns shown in Fig. $7 \mathrm{~b}$ and on the right side of Fig. 8b. The orange colour indicates the intensity distribution for the projected pattern in Fig. $7 \mathrm{~b}$ and the blue one indicates the intensity distribution for the extracted pattern in Fig. 8b. The regularity of the pattern intensity distribution like that shown in Fig. 8c is largely restored, but slight modifications are still needed. For example, the third and fifth intensity relations are not restored, and the eighth and tenth are over sensitive.

The next processing uses a method for correcting the pattern intensities in the frequency domain. Using a Fast Fourier Transform (FFT) to convert Fig. 8b to a frequency region, we obtain a power spectral image like that shown in Fig. 9a. Filtering Fig. 9a with Equation (13) leaves a power spectrum like that shown in Fig. 9b. Using Fig. 9b for inverse FFT gives the re-corrected pattern image $I_{(5)}$ shown in Fig. 9c.

Finally, a comprehensive analysis is performed of the images of the experimental results.

First, for each stripe shown in Fig. 9c we calculate the stripe intensity as the average pixel intensity and find the re-corrected pattern intensity distribution.

Fig. 10 compares the projected pattern, the re-corrected pattern, the corrected pattern for the two captured images and the stripe intensity distribution for the uncorrected extracted pattern. The orange line in the graph is the stripe intensity distribution for the projected pattern image in Fig. 7b, the black one is the stripe intensity distribution for the re-corrected pattern in Fig. 9c, the yellow one is the stripe intensity distribution for the two captured images (the result of dividing Figs 7c and 7a), and gray shows the stripe intensity distribution for the uncorrected extracted pattern in Fig. 7d. The order of stripes is from the bottom of the image. Fig. 10 shows that the intensity distribution of the corrected pattern is more similar than the uncorrected pattern to that of the projected pattern.

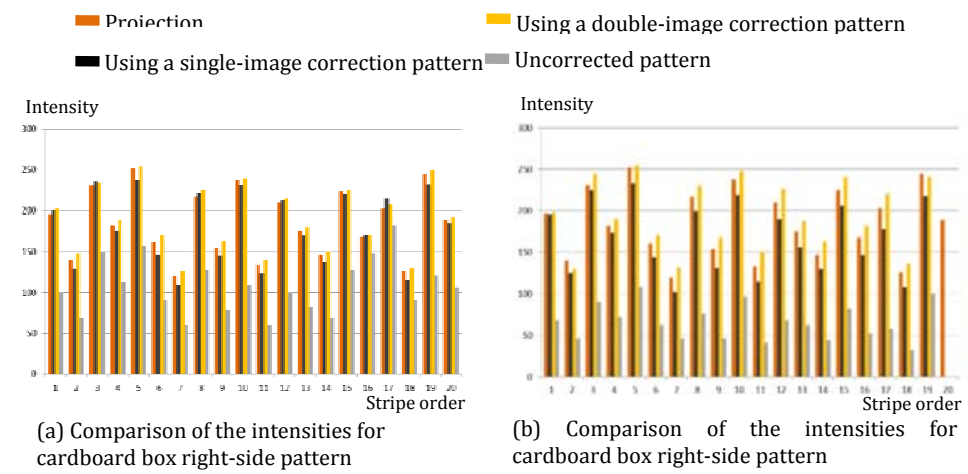

Fig. 10. Comparison of the pattern intensity distributions in the cardboard box experiment 
The evaluation method is next introduced for the intensity distribution correction effect of the observed pattern stripes in this study. The goal of correction is to match to the possible extent, the intensity of the observed pattern stripes with that of the corresponding projected pattern. However, because it is difficult to create a one-to-one intensity correspondence, the correction effect can instead be evaluated as the ratio of the absolute difference of the intensity value of the corrected stripe intensity and the corresponding projected stripe with the intensity of the projected stripe. The ratio and the error rate are then defined. The error rate is $0 \%$ in the case where the intensities are fully reconstructed. Otherwise, a correspondence relation detection method from the references is used for the determination. The average error rate is the average intensity of each stripe in the projected pattern, minus the average of the absolute difference of the average intensity in each observed pattern stripe and the average value of each stripe in the projected pattern. The maximum error rate is the largest error value among the calculations. The minimum error rate is the smallest error value among the calculations.

Regarding the results of the experiment for the box combination, the average intensity in the projected pattern was 185 for the pattern in the right image, the average intensity of the pattern re-corrected by the proposed method was 179 , and the average error rate was $3 \%$. The maximum error rate was $8 \%$, and the minimum error rate was $1 \%$. The average intensity for the uncorrected extracted pattern was 106 , and the average error rate was $42 \%$. The maximum error rate was $53 \%$, and the minimum error rate was $10 \%$.

In the case of correction by the two acquired images shown in Figs 7a and c, the average intensity in the corrected pattern was 189 , and the average error rate was $2 \%$. The maximum error rate was $6 \%$, and the minimum error rate was less than $1 \%$.

For the left pattern, the average intensity of the projected pattern was 185 , the average intensity of the corrected pattern was 169 , and the average error rate was $8 \%$. The maximum error rate was $14 \%$, and the minimum error rate was less than $1 \%$. In the uncorrected case, the average intensity of the extracted pattern was 67 , and the average error was $63 \%$. The maximum error rate was $73 \%$, and the minimum error rate was $55 \%$.

In the case of intensity correction by division, using the two captured images shown in Fig. 7a and c, the average intensity for the corrected pattern was 195, and the average error rate was $5 \%$. The maximum error rate for the corresponding stripes between the corrected pattern and the projected pattern was $10 \%$, and the minimum error rate was $1 \%$.

The above results indicate that the proposed method significantly improves the approximation of the projected pattern by the intensity distribution of the observed pattern. The average error rate for the re-corrected pattern and the projected pattern is kept within $10 \%$, and the maximum error rate within $15 \%$. A detection experiment was also performed, using a method from the literature to detect the correspondence relations using the corrected pattern [14], the uncorrected extracted pattern and the two acquired images. Only around half of the stripes were detected 
before correction, but after application of the method proposed, the detection rate was $100 \%$. Table 1 summarizes the detection results and the effects of stripe correction.

To verify the efficiency and applicability of the proposed method, measurement experiments were also performed using head models (Fig. 11a) and cylinders (Fig. 11b) in addition to the cardboard boxes. These experiments respectively used 30 and 40 stripes in the optimal intensity modulation patterns shown in Fig. 11c and d. These experiments were performed with both stationary and moving measured objects. Table 3 summarizes the results of the experiments. As Table 2 shows, the average stripe detection rate was less than $60 \%$ before the intensity correction, but reached $100 \%$ using the proposed method.

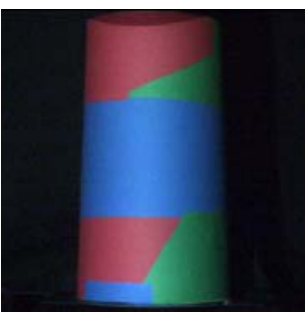

(a) Cylinder

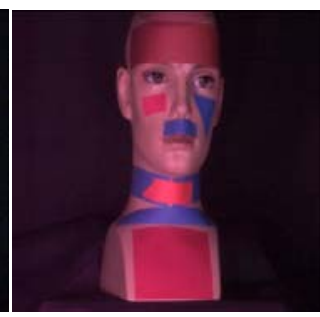

(b) Mannequin
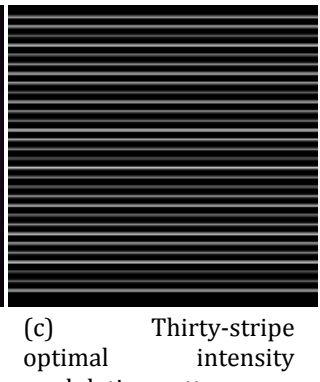
optimal intensity
modulation pattern

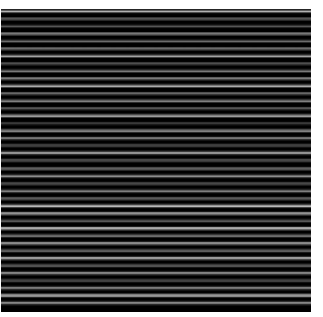

(d) Forty-stripe optimal intensity modulation

Fig. 11. Measured objects and projection patterns

Table 1. Pattern intensity correction effects in the cardboard box experiment

\begin{tabular}{|c|c|c|c|c|}
\hline \multicolumn{5}{|c|}{ (a) Right-side pattern } \\
\hline & Image & Avgerage error & Max error & Min error \\
\hline Single in & (no intensity correction) & $42 \%$ & $53 \%$ & $10 \%$ \\
\hline Double & $\begin{array}{l}\text { ge intensity correction } \\
\text {-image method) }\end{array}$ & $2 \%$ & $6 \%$ & $1 \%$ \\
\hline Single & $\begin{array}{l}\text { ge intensity correction } \\
\text { posed method) }\end{array}$ & $3 \%$ & $8 \%$ & $1 \%$ \\
\hline \multicolumn{5}{|c|}{ (b) Left-side pattern } \\
\hline & Image & Avgerage error & Max error & Min error \\
\hline Single in & (no intensity correction) & $63 \%$ & $73 \%$ & $55 \%$ \\
\hline Double & $\begin{array}{l}\text { ge intensity correction } \\
\text {-image method) }\end{array}$ & $5 \%$ & $10 \%$ & $1 \%$ \\
\hline Single- & $\begin{array}{l}\text { ge intensity correction } \\
\text { posed method) }\end{array}$ & $8 \%$ & $14 \%$ & $1 \%$ \\
\hline \multicolumn{5}{|c|}{ (c) Results of pattern detection in the cylinder and mannequin experiments } \\
\hline \multirow{2}{*}{\multicolumn{3}{|c|}{ Measured object }} & \multicolumn{2}{|c|}{ Cardboard box } \\
\hline & & & Left & Right \\
\hline \multicolumn{3}{|c|}{ Projected stripe count } & 19 & 20 \\
\hline \multirow{3}{*}{$\begin{array}{l}\text { Detecti- } \\
\text { on }\end{array}$} & \multicolumn{2}{|c|}{$\begin{array}{c}\text { Stripes detected by a single image } \\
\text { Detection rate (no intensity correction) }\end{array}$} & $\begin{array}{c}11 \\
58 \%\end{array}$ & $\begin{array}{c}10 \\
50 \%\end{array}$ \\
\hline & \multicolumn{2}{|c|}{$\begin{array}{c}\text { Stripes detected by two images } \\
\text { Detection rate (two images acquired) }\end{array}$} & $\begin{array}{c}19 \\
100 \%\end{array}$ & $\begin{array}{c}20 \\
100 \%\end{array}$ \\
\hline & \multicolumn{2}{|c|}{$\begin{array}{l}\text { Stripes detected by a single image } \\
\text { Detection rate (proposed method) }\end{array}$} & $\begin{array}{c}19 \\
100 \%\end{array}$ & $\begin{array}{c}20 \\
100 \%\end{array}$ \\
\hline
\end{tabular}


Table 2. Pattern detection in the cylinder and mannequin experiments

\begin{tabular}{|c|c|c|c|c|}
\hline Measured object & Mannequin & \multicolumn{3}{|c|}{ Cylinder } \\
\hline Projected stripe count & 20 & 20 & 30 & 40 \\
\hline Stripes detected by a single image & 9 & 14 & 22 & 21 \\
Detection rate (no intensity correction) & $45 \%$ & $70 \%$ & $73 \%$ & $53 \%$ \\
\hline Stripes detected by two images & 20 & 20 & 30 & 40 \\
Detection rate (two images acquired) & $100 \%$ & $100 \%$ & $100 \%$ & $100 \%$ \\
\hline Stripes detected by a single image & 20 & 20 & 30 & 40 \\
Detection rate (proposed method) & $100 \%$ & $100 \%$ & $100 \%$ & $100 \%$ \\
\hline
\end{tabular}

\section{Conclusions}

This study proposes a method for three-dimensional measurement of an object having a complex colour distribution from a single observation image. The experimental results show that the object measurement using optimal intensity modulation patterns with 20,30 and 40 lines detected the observed pattern with less than $60 \%$ accuracy before the correction, but after intensity correction following the proposed method, the detection rate reached $100 \%$. This also indicates that threedimensional measurements are possible using only a single image. In the future research we will look for ways to expand the application scope of the proposed method.

\section{References}

1. Lu, C., H. Ka mitomo, K. Sun, K. Tsujino, G. Cho. A Three-Dimensional Camera: Development and Applications of a Three-Dimensional Image Measurement System. - IEEJ Trans. ElS, Vol. 131, 2011, No 2, pp. 320-328.

2. Cho, G., C. Lu. Practical 3-D Measurement System Based on Projection Pattern Control Techniques. - IEEJ Trans. EIS, Vol. 127, 2007, No 4, pp. 561-567.

3. L u, C., L. X i a n g. Optimal Intensity-Modulation Projection Technique for Three-Dimensional Shape Measurement. - Applied Optics, Vol. 42, 2003, No 23, pp. 4649-4657.

4. L u, C., S. I n o k u c h i. Intensity-Modulated Moiré Topography. - Applied Optics, Vol. 38, 1999, No 19, pp. 4019-4029.

5. L u, C., G. C h o. 3-D Image Measurement by Combination of Monochrome-Projection ColourAnalysis and OIMP Technique. - Transactions of the Institute of Systems, Control and Information Engineers, Vol. 17, 2006, No 6, pp. 233-240.

6. L u, C., G. Cho, J. Zh a o. Practical 3-D Image Measurement System Using MonochromeProjection Colour-Analysis Technique. - In: Proc. of 7th IASTED International Conference on Computer Graphics and Imaging, Kauai, Hawaii, USA, 2004, pp. 266-271.

7. Gevers, T., A. S m e ulders. Colour-Based Object Recognition. - Pattern Recognition, Vol. 32, 1999, No 3, pp. 453-464.

8. B a j csy, R., S. W. Le e, A. Le on ardis. Detection of Diffuse and Specular Interface Reflections and Inter-Reflections by Colour Image Segmentation. - International Journal of Computer Vistion, Vol. 17, 1996, No 3, pp. 241-272.

9. Shafer, S. Using Colour to Separate Reflection Components. - Colour Research and Applications, Vol. 10, 1985, pp. 210-218.

10. S u n, K., C. L u. A High-Speed 3D Image Measurement Method. - Artificial Life and Robotics, Vol. 16, 2012, No 4, pp. 526-528.

11. S u n, K., C. L u. Stripes Extraction Technique of Projection Pattern in Frequency Domain for 3D Shape Measurement Based Pattern Projection Technique. - In: Proc. of 2009 International Conference on Optical Instrument and Technology (OIT'09), No 7513-126. 
12. S u n, K., C. L u. Stripes Extraction Technique of Projection Pattern for 3D Shape Measurement. In: International Symposium on Artificial Life and Robotics AROB 15th 10 Symposium 2010, No OS19-5, pp. 50-53.

13. Sun, K., H. Kamitomo, C. Lu. A High-Speed 3-D Image Measurement Method. In: International Symposium on Artificial Life and Robotics AROB 16th 11 Symposium 2011, No OS-131, p. 36.

14. L u, C., L. X i ang, S. In o k u ch i. Optimization of the Projection Pattern and a Quasi-Optimal Combination Generation Algorithm for 3-D Shape Measurement. - IEICE D-2, Vol. 88, 2005, No 2, pp. 325-333. 